Commun. Fac. Sci. Univ. Ank Ser. A1 Math. Stat.

Volume 68, Number 2, Pages 20372049(2019)

DOI: $10.31801 /$ cfsuasmas. 586057

ISSN 1303-5991 E-ISSN 2618-6470

http://communications.science.ankara.edu.tr/index.php?series=A1

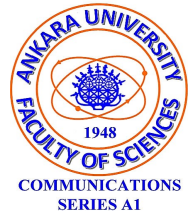

\title{
A REGRESSION TYPE ESTIMATOR FOR MEAN ESTIMATION UNDER RANKED SET SAMPLING ALONGSIDE THE SENSITIVITY ISSUE
}

\author{
USMAN SHAHZAD, MUHAMMAD HANIF, NURSEL KOYUNCU, \\ AND AMELIA VICTORIA GARCIA LUENGO
}

\begin{abstract}
Koyuncu and Kadilar 7 introduced a family of estimators under simple random sampling. In this article; we adapt these estimators for ranked set sampling. Further, we suggest a regression-type estimator of population mean utilizing available supplementary information under ranked set sampling scheme alongside the sensitivity issue when the variate of interest is sensitive. The bias and mean square error of the suggested estimator is determined theoretically for both situations. A simulation study has been done to demonstrate the percentage relative efficiency of proposed estimators over the adapted and reviewed estimators.
\end{abstract}

\section{INTRODUCTION}

In sampling survey, the supplementary information is mostly utilized to enhance precision of the estimators due to correlation between the study and the supplementary variables. Many authors including Sisodia and Dwivedi [17, Singh and Kakran [14, Upadhyaya and Singh [20, Tailor and Sharma [19, Koyuncu and Kadilar 7 and Shahzad [13 have developed some estimators for estimation of the population mean $\bar{Y}$ under simple random sampling (SRS) scheme. But consider a genuine circumstances where the study variate can't be effectively measured or is excessively costly, making it impossible to do as such, however can be ranked effectively at no cost or extremely little cost. It is realized that the estimate of the population mean utilizing ranked set sampling (RSS) is more productive than the one acquired utilizing SRS.

McIntyre 8 introduced the concept of RSS. Many authors such as Samawi and Muttlak [12, Bouza 1, Kadilar et al. [5] and Mehta and Mandowara 9] use judgmental RSS where ranking is done with respect to auxiliary variable $X$. Taking

Received by the editors: February 09, 2018; Accepted: June 14, 2019.

2010 Mathematics Subject Classification. Primary 05C38, 15A15; Secondary 05A15, 15A18.

Key words and phrases. Mean squared error, ranked set sampling, sensitivity, percentage relative efficiency.

(C)2019 Ankara University Communications Faculty of Sciences University of Ankara-Series A1 Mathematics and Statistics 
motivation from these we shall adapt Koyuncu and Kadilar [7] family of estimators under RSS. We shall also propose a new regression type estimator for the estimation of mean. Further, we shall discussed all the reviewed, adapted and proposed estimators for the scramble response utilizing different scrambled response models in upcoming sections.

In RSS scheme, $m$ independent random set, each of size $m$ are selected with equal probability and with replacement from the population. The units of each random set are ranked with respect to the characteristic of the study variable or auxiliary variable. Then the smallest unit is selected from the first ordered set and second smallest unit is selected from the second ordered and it is continued until the unit with largest value is chosen from the $m$ th set. This cycle maybe repeated $r$ times, so $m r=n$ units have been measured during this process. In this paper we assumed that we rank on the auxiliary variable, let $\left(y_{[i]}, x_{(i)}\right)$ denote $i$ th judgment ordering in the $i$ th set for the study variable and $i$ th set for the auxiliary variable. Further let $\bar{y}_{[n]}=\frac{1}{n} \sum_{i=1}^{n} y_{[i]}, \bar{x}_{(n)}=\frac{1}{n} \sum_{i=1}^{n} x_{(i)}$ are the sample means under RSS and $\bar{Y}, \bar{X}$ are population means for the study and auxiliary variables, respectively

To obtain the bias and mean squared error (MSE) expressions, let us define $\bar{y}_{[n]}=\left(1+\mathfrak{e}_{o}\right) \bar{Y}, \bar{x}_{(n)}=\left(1+\mathfrak{e}_{1}\right) \bar{X}$. The expectations of $e$ terms can be written as follows:

$E\left(\mathfrak{e}_{o}\right)=E\left(\mathfrak{e}_{1}\right)=0$

$E\left(\mathfrak{e}_{o}^{2}\right)=\lambda C_{y}^{2}-W_{y_{[i]}}^{2}=\mathfrak{c}_{0}$,

$E\left(\mathfrak{e}_{1}^{2}\right)=\lambda C_{x}^{2}-W_{x_{(i)}}^{2}=\mathfrak{c}_{1}$,

$E\left(\mathfrak{e}_{o} \mathfrak{e}_{1}\right)=\lambda \rho C_{y} C_{x}-W_{y x_{(i)}}=\mathfrak{c}_{01}$.

where

$W_{y_{[i]}}^{2}=\frac{1}{m^{2} r \bar{Y}^{2}} \sum_{i=1}^{m} \tau_{y[i]}^{2}, W_{x_{(i)}}^{2}=\frac{1}{m^{2} r \bar{X}^{2}} \sum_{i=1}^{m} \tau_{x(i)}^{2}, W_{y x_{(i)}}=\frac{1}{m^{2} r \bar{X} \bar{Y}} \sum_{i=1}^{m} \tau_{y x(i)}, \lambda=\frac{1}{n}$.

Further $\tau_{y x(i)}=\left(\mu_{y[i]}-\bar{Y}\right)\left(\mu_{x(i)}-\bar{X}\right), \tau_{x(i)}=\left(\mu_{x(i)}-\bar{X}\right), \tau_{y[i]}=\left(\mu_{y[i]}-\bar{Y}\right)$. Note that $\left(\mu_{y[i]}, \mu_{x(i)}\right)$ belongs to some specific distribution, depends upon order statistic.

The traditional ratio estimator in RSS is given by

$$
\hat{\mathfrak{t}}_{\text {R.rss }}=\bar{y}_{[n]}\left[\frac{\bar{X}}{\bar{x}_{(n)}}\right] \text {. }
$$

The (MSE) of $\hat{\mathfrak{t}}_{\text {R.rss }}$ is

$$
M S E\left(\hat{\mathfrak{t}}_{R . r s s}\right)=\bar{Y}^{2}\left[\mathfrak{c}_{0}+\mathfrak{c}_{1}-2 \mathfrak{c}_{01}\right] .
$$

Mehta and Mandowara 9] adapted Sisodia and Dwivedi [17, Singh and Kakran [14], Upadhyaya and Singh [20] estimators under RSS as follows

$$
\hat{\mathfrak{t}}_{S D . r s s}=\bar{y}_{[n]}\left[\frac{\bar{X}+C_{x}}{\bar{x}_{(n)}+C_{x}}\right] \text {, }
$$




$$
\begin{gathered}
\hat{\mathfrak{t}}_{S K . r s s}=\bar{y}_{[n]}\left[\frac{\bar{X}+\beta_{2}(x)}{\bar{x}_{(n)}+\beta_{2}(x)}\right], \\
\hat{\mathfrak{t}}_{U P 1 . r s s}=\bar{y}_{[n]}\left[\frac{\bar{X} C_{x}+\beta_{2}(x)}{\bar{x}_{(n)} C_{x}+\beta_{2}(x)}\right], \\
\hat{\mathfrak{t}}_{U P 2 . r s s}=\bar{y}_{[n]}\left[\frac{\bar{x}_{(n)} C_{x}+\beta_{2}(x)}{\bar{X} C_{x}+\beta_{2}(x)}\right] .
\end{gathered}
$$

The MSEs' of these estimators are as follows

$$
\begin{gathered}
M S E\left(\hat{\mathfrak{t}}_{S D . r s s}\right)=\bar{Y}^{2}\left[\delta_{S D . r s s}^{2} \mathfrak{c}_{0}+\mathfrak{c}_{1}-2 \delta_{S D . r s s} \mathfrak{c}_{01}\right], \\
M S E\left(\hat{\mathfrak{t}}_{S K . r s s}\right)=\bar{Y}^{2}\left[\delta_{S K . r s s}^{2} \mathfrak{c}_{0}+\mathfrak{c}_{1}-2 \delta_{S K . r s s} \mathfrak{c}_{01}\right], \\
M S E\left(\hat{\mathfrak{t}}_{U P 1 . r s s}\right)=\bar{Y}^{2}\left[\delta_{U P 1 . r s s}^{2} \mathfrak{c}_{0}+\mathfrak{c}_{1}-2 \delta_{U P 1 . r s s} \mathfrak{c}_{01}\right], \\
M S E\left(\hat{\mathfrak{t}}_{U P 2 . r s s}\right)=\bar{Y}^{2}\left[\delta_{U P 2 . r s s}^{2} \mathfrak{c}_{0}+\mathfrak{c}_{1}+2 \delta_{U P 2 . r s s} \mathfrak{c}_{01}\right]
\end{gathered}
$$

where $\delta_{S D . r s s}=\frac{\bar{X}}{\bar{X}+C_{x}}, \delta_{S K . r s s}=\frac{\bar{X}}{\bar{X}+\beta_{2}(x)}, \delta_{U P 1 . r s s}=\delta_{U P 2 . r s s}=\frac{\bar{X} C_{x}}{\bar{X} C_{x}+\beta_{2}(x)}$.

The usual difference type estimator in RSS is given by

$$
\hat{\mathfrak{t}}_{D . r s s}=\bar{y}_{[n]}+d\left(\bar{X}-\bar{x}_{(n)}\right) .
$$

The MSE of $\hat{\mathbf{t}}_{\text {D.rss }}$ is

$$
\operatorname{MSE}\left(\hat{\hat{\mathfrak{t}}_{\text {D.rss }}}\right)=\bar{Y}^{2} \mathfrak{c}_{0}+d^{2} \bar{X}^{2} \mathfrak{c}_{1}-2 \bar{X} \bar{Y} d \mathfrak{c}_{01} .
$$

The optimum value of $d$ is

$$
d^{o p t}=\frac{\bar{Y} \mathfrak{c}_{01}}{\bar{X} \mathfrak{c}_{1}}
$$

Hence the minimum MSE after utilizing $d^{\text {opt }}$ is as follows

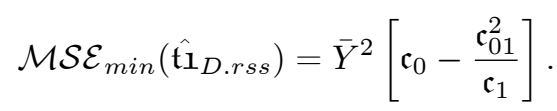

The $\mathcal{M S \mathcal { E } _ { \text { min } }}\left(\hat{\mathbf{t}}_{\text {D.rss }}\right)$ is similar to MSE of regression estimator i.e. $\hat{\mathfrak{t}}_{\text {Reg.rss }}=$ $\bar{y}_{[n]}+\hat{\beta}\left(\bar{X}-\bar{x}_{(n)}\right)$. Taking motivation from Tailor and Sharma [19, Mehta and Mandowara [9] also introduced an estimator under RSS scheme. The minimum MSE of their estimator was equivalent to $\mathcal{M S E}_{\min }\left(\hat{\mathfrak{t}}_{D . r s s}\right)$. Note that when $d^{\text {opt }}$ is unknown we can use sample regression coefficient i.e. $\hat{\beta}=\frac{\lambda\left(\hat{C}_{y x}-\hat{W}_{y x_{(i)}}\right) \hat{R}}{\left(\hat{C}_{x}^{2}-\hat{W}_{x_{(i)}}^{2}\right)}$; see Singh et al. [15], where $\hat{R}=\frac{\bar{y}_{[n]}}{\bar{x}_{(n)}}$. 


\section{Adapted family of estimators under RSS}

We adapt Koyuncu and Kadilar [7] family of estimators to RSS scheme as follows

$$
\hat{\mathbf{t}}_{k k . r s s}=w \bar{y}_{[n]}\left[\frac{(\bar{X} a+b)}{\alpha\left(\bar{x}_{(n)} a+b\right)+(1-\alpha)(\bar{X} a+b)}\right]^{g}
$$

where $a(\neq 0), b$ are either real numbers of functions of the known parameters of the auxiliary variable and $g, \alpha, w$ are suitable chosen scalars such that the MSE of $\hat{\mathfrak{t}}_{\mathbf{I}_{k k . s s}}$ is minimum. We can generate some new estimators from $\hat{\mathfrak{t}}_{\text {lk.rss }}$ using suitable scalars as given in Table1.

TABLE 1 . Family members of $\hat{\mathfrak{t}}_{k k . r s s}$ for $(\alpha=1)$

\begin{tabular}{|c|c|c|c|}
\hline Est. & & $\mathrm{a}$ & $\mathrm{b}$ \\
\hline $\mathfrak{t}_{\mathbf{l}_{k k} r s_{1}}=w \bar{y}_{[n]}$ & $X$ & 1 & 0 \\
\hline 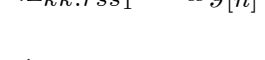 & {$\left[\bar{x}_{(n)}\right]$} & & \\
\hline$\hat{\mathfrak{t}}_{k k . r s s_{2}}=w \bar{y}_{[n]}$ & $\frac{X+C_{x}}{\bar{x}_{(n)}+C_{x}}$ & 1 & $C_{x}$ \\
\hline$\hat{\mathfrak{t}}_{k k . r s s_{3}}=w \bar{y}_{[n]}$ & {$\left[\frac{\bar{X} \beta_{2}(x)+C_{x}}{\bar{x}_{(n)} \beta_{2}(x)+C_{x}}\right.$} & $\beta_{2}(x)$ & $C_{x}$ \\
\hline$\hat{\mathfrak{t}}_{\mathbf{l}_{k k . r s s_{4}}}=w \bar{y}_{[n]}$ & {$\left[\frac{\bar{X} C_{x}+\beta_{2}(x)}{\bar{x}_{(n)} C_{x}+\beta_{2}(x)}\right.$} & $C_{x}$ & $\beta_{2}(x)$ \\
\hline$\hat{\mathfrak{t}}_{k k . r s s_{5}}=w \bar{y}_{[n]}$ & {$\left[\frac{X \beta_{1}(x)+S_{x}}{\bar{x}_{(n)} \beta_{1}(x)+S_{x}}\right.$} & $\beta_{1}(x)$ & $S_{x}$ \\
\hline$\hat{\mathfrak{t}}_{{ }_{k k . r s s_{6}}}=w \bar{y}_{[n]}$ & $\frac{X \beta_{2}(x)+S_{x}}{\bar{x}_{(n)} \beta_{2}(x)+S_{x}}$ & $\beta_{2}(x)$ & $S_{x}$ \\
\hline$\hat{\mathfrak{t}}_{k k . r s s_{7}}=w \bar{y}_{[n]}$ & {$\left[\frac{X+\rho}{\bar{x}_{(\underline{n})}+\rho}\right\rfloor$} & 1 & $\rho$ \\
\hline$\hat{\mathfrak{t}}_{\mathbf{l}_{k k . r s s_{8}}}=w \bar{y}_{[n]}$ & {$\left[\frac{X+\beta_{2}(x)}{\bar{x}_{(n)}+\beta_{2}(x)}\right.$} & 1 & $\beta_{2}(x)$ \\
\hline
\end{tabular}

Note that Kadilar et al. [5] estimator is also a member of $\hat{\mathbf{t}}_{k k . r s s}$ family, denoted by $\hat{\mathfrak{t}}_{k k . r s s_{1}}$ in Table [1]. The MSE of $\hat{\mathfrak{t}}_{k k . r s s}$ is given by

$$
\mathcal{M S E}\left(\hat{\mathfrak{t}}_{k k . r s s}\right)=\bar{Y}^{2}+w^{2} \delta_{B_{k k}}-2 w \delta_{A_{k k}}
$$

where

$$
\begin{aligned}
& \delta_{A_{k k . r s s}}=\bar{Y}^{2}\left[1+\frac{g(g+1)}{2} \alpha^{2} \mathfrak{v}^{2} \mathfrak{c}_{1}-g \alpha \mathfrak{v} \mathfrak{c}_{o 1}\right], \\
& \delta_{B_{k k . r s s}}=\bar{Y}^{2}\left[1+\mathfrak{c}_{o}+\left(2 g^{2}+g\right) \alpha^{2} \mathfrak{v}^{2} \mathfrak{c}_{1}-4 g \alpha \mathfrak{v} \mathfrak{c}_{o 1}\right] .
\end{aligned}
$$


which is minimum for

$$
w^{o p t}=\frac{\delta_{A_{k k . r s s}}}{\delta_{B_{k k . r s s}}} .
$$

The minimum MSE of $\hat{\mathfrak{t}}_{\text {kk.rss }}$ is given by

$$
\mathcal{M S E}_{\text {min }}\left(\hat{\mathfrak{t}}_{k k . r s s}\right)=\left[\bar{Y}^{2}-\frac{\delta_{A_{k k . r s s}^{2}}}{\delta_{B_{k k . r s s}}}\right] .
$$

\section{Suggested REgREssion type estimator Under RSS}

We propose the following estimator under RSS as follows

$$
\hat{\mathfrak{t}}_{N}=w_{1}\left\{\bar{y}_{[n]}+\hat{\beta}\left(\bar{X}-\bar{x}_{(n)}\right)\right\}+w_{2}(\bar{X}-\bar{x}),
$$

where $w_{1}$ and $w_{2}$ are the two unknown weights. Expanding right hand side of $\hat{\mathbf{t}}_{N}$, we obtain:

$$
\hat{\mathfrak{t}}_{N}-\bar{Y}=w_{1} \bar{Y}\left\{1+\mathfrak{e}_{o}-R^{\prime} \hat{\beta} \mathfrak{e}_{1}\right\}-w_{2} \bar{X} \mathfrak{e}_{1}-\bar{Y} .
$$

The bias of $\hat{\mathfrak{t}}_{N}$ is

$$
B\left(\hat{\mathfrak{t}}_{N}\right)=\left(w_{1}-1\right) \bar{Y}
$$

The MSE of $\hat{\mathfrak{t}}_{N}$ is

$$
\mathcal{M S E}\left(\hat{\mathfrak{t}}_{N}\right)=\bar{Y}^{2}+w_{1}^{2} \delta_{A_{N}}+w_{2}^{2} \delta_{B_{N}}+2 w_{1} w_{2} \delta_{C_{N}}-2 w_{1} \delta_{D_{N}},
$$

where

$$
\begin{aligned}
\delta_{A_{N}} & =\bar{Y}^{2}\left[1+\mathfrak{c}_{o}+R^{\prime 2} \beta^{2} \mathfrak{c}_{1}-2 \beta^{2} R^{\prime} \mathfrak{c}_{o 1}\right], \\
\delta_{B_{N}} & =\bar{X}^{2} \mathfrak{c}_{1} \\
\delta_{C_{N}} & =\bar{X} \bar{Y}\left[\beta R^{\prime} \mathfrak{c}_{1}-\mathfrak{c}_{o 1}\right] \\
\delta_{D_{N}} & =\bar{Y}^{2} \\
R^{\prime} & =\frac{\bar{X}}{\bar{Y}}
\end{aligned}
$$

which is minimum for

$$
w_{1}^{o p t}=\left[\frac{\delta_{B_{N}} \delta_{D_{N}}}{\delta_{A_{N}} \delta_{B_{N}}-\delta_{C_{N}}^{2}}\right],
$$

and

$$
w_{2}^{o p t}=\left[-\frac{\delta_{C_{N}} \delta_{D_{N}}}{\delta_{A_{N}} \delta_{B_{N}}-\delta_{C_{N}}^{2}}\right] .
$$

Using optimum values, the minimum MSE of $\hat{\mathbf{t}}_{N}$ is given by

$$
\mathcal{M S E}_{\text {min }}\left(\hat{\mathbf{t}}_{N}\right)=\left[\bar{Y}^{2}-\frac{\delta_{B_{N}} \delta_{D_{N}}^{2}}{\delta_{A_{N}} \delta_{B_{N}}-\delta_{C_{N}}^{2}}\right] .
$$




\section{Sensitivity issue Under RSS}

The questions related to drug addiction, reckless driving, illegal income and indiscriminate gambling are known as sensitive questions. In these circumstances, we can't expect a truthful direct response from people. Many authors like Eichhron and Hayre [3], Pollock and Bek [10], Saha [1] and Diana and Perri [2] have studied the mean of a sensitive study variate without utilizing supplementary information. Further, Sousa et al. 18] introduced traditional mean per unit estimator for the case of scramble response. Gupta et al. 4 introduced traditional regression and generalized regression estimator assuming the problem of sensitivity. Koyuncu et al. [6] also done the work in this context by introducing exponential-type estimators.

Suppose $Z$ be the response of study variable $Y$. Than to obtain the MSE expressions, let us define $\bar{z}_{[n]}=\left(1+\mathfrak{e}_{z}\right) \bar{Z}$. Using these terms the expectaions are given by $E\left(\mathfrak{e}_{z}\right)=0$,

$E\left(\mathfrak{e}_{z}^{2}\right)=\lambda C_{z}^{2}-W_{z_{[i]}}^{2}=\mathfrak{c}_{z}$

$E\left(\mathfrak{e}_{z} \mathfrak{e}_{1}\right)=\lambda \rho_{z x} C_{z} C_{x}-W_{z x_{(i)}}=\mathfrak{c}_{z 1}$.

where $W_{z_{[i]}}^{2}=\frac{1}{m^{2} r \bar{Z}^{2}} \sum_{i=1}^{m} \tau_{z[i]}^{2}, W_{z x_{(i)}}=\frac{1}{m^{2} r \bar{X} \bar{Z}} \sum_{i=1}^{m} \tau_{z x(i)}, \lambda=\frac{1}{n}$.

Further $\tau_{z x(i)}=\left(\mu_{z[i]}-\bar{Z}\right)\left(\mu_{x(i)}-\bar{X}\right), \tau_{z[i]}=\left(\mu_{z[i]}-\bar{Z}\right)$.

The estimator $\hat{\mathfrak{t}}_{\text {R.rss }}$ for the case of scrambled response is given by

$$
\hat{\mathfrak{z}}_{\text {R.rss }}=\bar{z}_{[n]}\left[\frac{\bar{X}}{\bar{x}_{(n)}}\right] \text {. }
$$

The MSE of $\hat{\mathfrak{z}}$ R.rss is

$$
\operatorname{MSE}\left(\hat{\mathfrak{z}}_{\text {R.rss }}\right)=\bar{Z}^{2}\left[\mathfrak{c}_{z}+\mathfrak{c}_{1}-2 \mathfrak{c}_{z 1}\right] .
$$

Mehta and Mandowara [9] estimators for scramble response can be written as follows

$$
\begin{gathered}
\hat{\mathfrak{z}}_{S D . r s s}=\bar{z}_{[n]}\left[\frac{\bar{X}+C_{x}}{\bar{x}_{(n)}+C_{x}}\right], \\
\hat{\mathfrak{z}}_{S K . r s s}=\bar{z}_{[n]}\left[\frac{\bar{X}+\beta_{2}(x)}{\bar{x}_{(n)}+\beta_{2}(x)}\right], \\
\hat{\mathfrak{z}}_{U P 1 . r s s}=\bar{z}_{[n]}\left[\frac{\bar{X} C_{x}+\beta_{2}(x)}{\bar{x}_{(n)} C_{x}+\beta_{2}(x)}\right], \\
\hat{\mathfrak{z}} U P 2 . r s s=\bar{z}_{[n]}\left[\frac{\bar{x}_{(n)} C_{x}+\beta_{2}(x)}{\bar{X} C_{x}+\beta_{2}(x)}\right] .
\end{gathered}
$$

The MSEs' of these estimators are

$$
\begin{aligned}
& M S E\left(\hat{\mathfrak{z}}_{S D . r s s}\right)=\bar{Z}^{2}\left[\delta_{S D . r s s}^{2} \mathfrak{c}_{z}+\mathfrak{c}_{1}-2 \delta_{S D . r s s} \mathfrak{c}_{z 1}\right], \\
& M S E\left(\hat{\mathfrak{z}}_{S K . r s s}\right)=\bar{Z}^{2}\left[\delta_{S K . r s s}^{2} \mathfrak{c}_{z}+\mathfrak{c}_{1}-2 \delta_{S K . r s s} \mathfrak{c}_{z 1}\right],
\end{aligned}
$$




$$
\begin{aligned}
& M S E\left(\hat{\mathfrak{z}}_{U P 1 . r s s}\right)=\bar{Z}^{2}\left[\delta_{U P 1 . r s s}^{2} \mathfrak{c}_{z}+\mathfrak{c}_{1}-2 \delta_{U P 1 . r s s} \mathfrak{c}_{z 1}\right], \\
& M S E\left(\hat{\mathfrak{z}}_{U P 2 . r s s}\right)=\bar{Z}^{2}\left[\delta_{U P 2 . r s s}^{2} \mathfrak{c}_{z}+\mathfrak{c}_{1}+2 \delta_{U P 2 . r s s} \mathfrak{c}_{z 1}\right] .
\end{aligned}
$$

The difference-estimator in RSS is as follows

$$
\hat{\mathfrak{z}}_{D . r s s}=\bar{z}_{[n]}+d\left(\bar{X}-\bar{x}_{(n)}\right) .
$$

The MSE of $\hat{\mathfrak{z}}_{\text {D.rss }}$ is

$$
\operatorname{MSE}\left(\hat{\mathfrak{z}}_{D . r s s}\right)=\bar{Z}^{2} \mathfrak{c}_{z}+d^{2} \bar{X}^{2} \mathfrak{c}_{1}-2 \bar{X} \bar{Z} d \mathfrak{c}_{z 1} .
$$

The optimum value of $d$ is

$$
d^{\text {opt }}=\frac{\bar{Z} \mathfrak{c}_{z 1}}{\bar{X} \mathfrak{c}_{1}}
$$

Hence the minimum MSE after utilizing $d^{\text {opt }}$ is as follows

$$
\mathcal{M S E}_{\text {min }}\left(\hat{\mathfrak{z}}_{D . r s s}\right)=\bar{Z}^{2}\left[\mathfrak{c}_{z}-\frac{\mathfrak{c}_{z 1}^{2}}{\mathfrak{c}_{1}}\right] .
$$

4.1. Adapted family of estimators for scrambled response under RSS. The adapted family of estimators i.e. $\hat{\mathfrak{z}}_{k k \text {.rss }}$ for the scramble response is as follows:

$$
\hat{\mathfrak{z}}_{k k . r s s}=w \bar{z}_{[n]}\left[\frac{(\bar{X} a+b)}{\alpha\left(\bar{x}_{(n)} a+b\right)+(1-\alpha)(\bar{X} a+b)}\right]^{g} .
$$

The definitions of the parameters in $\hat{\mathfrak{z}}_{k k . r s s}$ are same with $\hat{\mathfrak{t}}_{k k . r s s}$. Also one can generate new estimators using suitable scalars from $\hat{\mathfrak{z}}_{k k . r s s}$ as given in Table2. The MSE of $\hat{\mathfrak{z}}_{k k . r s s}$ is given by

$$
\mathcal{M S E}\left(\hat{\mathfrak{z}}_{k k . r s s}\right)=\bar{Z}^{2}+w^{2} \delta_{B_{k k}}-2 w \delta_{A_{k k}},
$$

where

$$
\begin{aligned}
& \delta_{A_{k k . r s s}}=\bar{Z}^{2}\left[1+\frac{g(g+1)}{2} \alpha^{2} \mathfrak{v}^{2} \mathfrak{c}_{1}-g \alpha \mathfrak{v} \mathfrak{c}_{o 1}\right] \\
& \delta_{B_{k k . r s s}}=\bar{Z}^{2}\left[1+\mathfrak{c}_{z}+\left(2 g^{2}+g\right) \alpha^{2} \mathfrak{v}^{2} \mathfrak{c}_{1}-4 g \alpha \mathfrak{v} \mathfrak{c}_{o 1}\right]
\end{aligned}
$$

which is minimum for

$$
\begin{gathered}
w^{o p t}=\frac{\delta_{A_{k k . r s s}}}{\delta_{B_{k k . r s s}}} \\
\mathcal{M S E}_{\text {min }}\left(\hat{\mathfrak{z}}_{k k . r s s}\right)=\left[\bar{Z}^{2}-\frac{\delta_{A_{k k . r s s}^{2}}}{\delta_{B_{k k . r s s}}}\right] .
\end{gathered}
$$


TABLe 2. Family members of $\hat{\mathfrak{z}}_{k k . r s s}$ for $(\alpha=1)$

\begin{tabular}{|c|c|c|c|}
\hline Est. & & $\mathrm{a}$ & $\mathrm{b}$ \\
\hline$\hat{\mathfrak{z}}_{k k . r s s_{1}}=w \bar{z}_{[n]}$ & $X$ & 1 & 0 \\
\hline & $\begin{array}{l}{\left[\bar{x}_{(n)}\right]} \\
{[\bar{X}+C}\end{array}$ & & \\
\hline$\hat{\mathfrak{z}}_{k k . r s s_{2}}=w \bar{z}_{[n]}$ & {$\left[\frac{\Lambda+C_{x}}{\bar{x}_{(n)}+C_{x}}\right]$} & 1 & $C_{x}$ \\
\hline$\hat{\mathfrak{z}}_{k k . r s s_{3}}=w \bar{z}_{[n]}$ & {$\left[\frac{X \beta_{2}(x)+C_{x}}{\bar{x}_{(n)} \beta_{2}(x)+C_{x}}\right]$} & $\beta_{2}(x)$ & $C_{x}$ \\
\hline$\hat{\mathfrak{z}}_{k k . r s s_{4}}=w \bar{z}_{[n]}$ & {$\left[\frac{X C_{x}+\beta_{2}(x)}{\bar{x}_{(n)} C_{x}+\beta_{2}(x)}\right]$} & $C_{x}$ & $\beta_{2}(x)$ \\
\hline$\hat{\mathfrak{z}}_{k k . r s s_{5}}=w \bar{z}_{[n]}$ & $\frac{X \beta_{1}(x)+S_{x}}{\bar{x}_{(n)} \beta_{1}(x)+S_{x}}$ & $\beta_{1}(x)$ & $S_{x}$ \\
\hline$\hat{\mathfrak{z}}_{k k . r s s_{6}}=w \bar{z}_{[n]}$ & {$\left[\frac{X \beta_{2}(x)+S_{x}}{\bar{x}_{(\underline{n})} \beta_{2}(x)+S_{x}}\right]$} & $\beta_{2}(x)$ & $S_{x}$ \\
\hline$\hat{\mathfrak{z}}_{k k . r s s_{7}}=w \bar{z}_{[n]}$ & {$\left[\overline{\bar{x}}\left(\frac{n)}{\bar{X}}+\rho\right]\right.$} & 1 & $\rho$ \\
\hline$\hat{\mathfrak{z}}_{k k . r s s_{8}}=w \bar{z}_{[n]}$ & $\frac{X+\beta_{2}(x)}{\bar{x}_{(n)}+\beta_{2}(x)}$ & 1 & $\beta_{2}(x)$ \\
\hline
\end{tabular}

4.2. Suggested regression type estimator for scrambled response under RSS. The proposed estimator for the scramble response under RSS is given by

$$
\hat{\mathfrak{z}}_{N}=w_{1}\left\{\bar{z}_{[n]}+\hat{\beta}\left(\bar{X}-\bar{x}_{(n)}\right)\right\}+w_{2}(\bar{X}-\bar{x}) .
$$

The bias of $\hat{\mathfrak{z}}_{N}$ is

$$
B\left(\hat{\mathfrak{z}}_{N}\right)=\left(w_{1}-1\right) \bar{Z} .
$$

The MSE of $\hat{\mathfrak{z}}_{N}$ is given by

$$
\mathcal{M S E}\left(\hat{\mathfrak{z}}_{N}\right)=\bar{Z}^{2}+w_{1}^{2} \delta_{A_{N}}+w_{2}^{2} \delta_{B_{N}}+2 w_{1} w_{2} \delta_{C_{N}}-2 w_{1} \delta_{D_{N}},
$$

where

$$
\begin{aligned}
\delta_{A_{N}} & =\bar{Z}^{2}\left[1+\mathfrak{c}_{z}+R^{\prime \prime 2} \beta^{2} \mathfrak{c}_{1}-2 \beta^{2} R^{\prime \prime} \mathfrak{c}_{o 1}\right], \\
\delta_{B_{N}} & =\bar{X}^{2} \mathfrak{c}_{1} \\
\delta_{C_{N}} & =\bar{X} \bar{Z}\left[\beta R^{\prime \prime} \mathfrak{c}_{1}-\mathfrak{c}_{o 1}\right] \\
\delta_{D_{N}} & =\bar{Z}^{2} \\
R^{\prime \prime} & =\frac{\bar{X}}{\bar{Z}}
\end{aligned}
$$


which is minimum for

and

$$
w_{1}^{o p t}=\left[\frac{\delta_{B_{N}} \delta_{D_{N}}}{\delta_{A_{N}} \delta_{B_{N}}-\delta_{C_{N}}^{2}}\right],
$$

$$
w_{2}^{o p t}=\left[-\frac{\delta_{C_{N}} \delta_{D_{N}}}{\delta_{A_{N}} \delta_{B_{N}}-\delta_{C_{N}}^{2}}\right] .
$$

Finally, using optimum values we obtain minimum MSE as follows

$$
\mathcal{M S E}_{\text {min }}\left(\hat{\mathfrak{z}}_{N}\right)=\left[\bar{Z}^{2}-\frac{\delta_{B_{N}} \delta_{D_{N}}^{2}}{\delta_{A_{N}} \delta_{B_{N}}-\delta_{C_{N}}^{2}}\right] .
$$

\section{Simulation Study}

We use a real data set for simulation study available in Singh [16]. This data set is already used by Mehta and Mandowara [9. The data consists of agricultural loans of all operating banks in different states of USA in 1997, where

$Y$ : Real estate farm loans in 1997 in USA \&

$X$ : Non-real estate farm loans in 1997 in USA.

Note that in our simulation procedure we adapt Singh et al. 15 for the calculation of $W_{y_{[i]}}^{2}, W_{x_{(i)}}^{2}$ and $W_{y x_{(i)}}$, where

$$
\begin{gathered}
W_{y_{[i]}}^{2}=\frac{1}{m^{2} r} \sum_{i=1}^{m}(R D Y[i]-1)^{2}, \\
W_{x_{(i)}}^{2}=\frac{1}{m^{2} r} \sum_{i=1}^{m}(R D X(i)-1)^{2}, \\
W_{y x_{(i)}}=\frac{1}{m^{2} r} \sum_{i=1}^{m}(R D Y[i]-1)(R D X(i)-1) .
\end{gathered}
$$

The expressions of $R D Y[i]$ are calculated as follows

$$
R D Y[1]=.25+.08 e_{1}, R D Y[2]=.50+.08 e_{2}, R D Y[3]=1.00+.08 e_{3} .
$$

The expressions of $R D X(i)$ are calculated as follows

$$
R D X(1)=.25+.05 e_{1}, R D X(2)=.50+.05 e_{2}, R D X(3)=1.00+.05 e_{3} .
$$

Further, $e_{i}$ for $(i=1,2,3)$ is normally distributed with zero mean and unit variance.

The steps of simulation the estimation of non-sensitive study variate are as follows

- Select a ranked set sample of size $n=12$ using $m=3$ distinct sets and $r=4$ cycles from the population containing $N$ units.

- Estimate mean $\hat{y}$ (say) through RSS.

- The whole process is repeated 3,000 times. 
- After that average MSE is calculated using $M S E=\frac{\sum_{i=1}^{3000}(\hat{y}-\bar{Y})^{2}}{3000}$.

- Finally, percent relative efficiency (PRE) is calculated as

$$
\operatorname{PRE}(.)=\frac{M S E\left(\hat{\mathfrak{t}}_{D . r s s}\right)}{M S E(.)} \times 100 .
$$

For the case of sensitivity, we use

Pollock and Beck [10] model i.e. $Z=Y+S_{2}$.

Eichhorn and Hayre [3] model i.e. $Z=S_{1} Y$.

Saha [11] model i.e. $Z=S_{1}\left(Y+S_{2}\right)$.

Diana and Perri 2 model i.e. $Z=\phi\left(Y+S_{2}\right)+(1-\phi) S_{1} Y$ where $\phi$ ranges $[0,1]$. In these models $S_{1}$ and $S_{2}$ are the scrambling variates. We assume that $S_{1}$ and $S_{2}$ are uniformly distributed i.e. $U(0,1)$. Now we perform the above mentioned simulation procedure for the scramble response by replacing non-sensitive study variable $Y$ with the sensitive study variable i.e. $Z$. Note that all the above mentioned scrambled-response models are applied one by one for the estimation of sensitive study variable.

TABLE 3. PREs for non-sensitive study variate

\begin{tabular}{|c|c|c|c|c|c|}
\hline Est. & $\mathrm{PRE}$ & Est. & $\mathrm{PRE}$ & Est. & $\mathrm{PRE}$ \\
\hline$\hat{\mathfrak{t}}_{\text {R.rss }}$ & 496.82 & $\hat{\mathfrak{t}}_{\text {D.rss }}$ & 100 & $\hat{\mathfrak{t}}_{\mathbf{I}_{k k . r s s 5}}$ & 2340.71 \\
\hline$\hat{\mathfrak{t}}_{S D . r s s}$ & 501.36 & $\hat{\mathfrak{t}}_{k k . r s s 1}$ & 641.99 & $\hat{\mathfrak{t}}_{k k . r s s 6}$ & 1647.31 \\
\hline$\hat{\mathfrak{t}}_{S K . r s s}$ & 513.45 & $\hat{\mathbf{t}}_{k k . r s s 2}$ & 647.54 & $\hat{\mathfrak{t}}_{k k . r s s 7}$ & 644.98 \\
\hline$\hat{\mathfrak{t}}_{U P . r s s 1}$ & 510.29 & $\hat{\mathbf{t}}_{k k . r s s 3}$ & 643.21 & $\hat{\mathfrak{t}}_{k k . r s s 8}$ & 662.28 \\
\hline$\hat{\mathbf{t}}_{U P . r s s 2}$ & 497.82 & $\hat{\mathfrak{t}}_{\text {l }}{ }_{k k r s s 4}$ & 658.42 & $\hat{\mathbf{t}}_{N}$ & 4812.21 \\
\hline
\end{tabular}

TABle 4. PREs for sensitive study variate using Pollock and Bek model (1976)

\begin{tabular}{|c|c|c|c|c|c|}
\hline Est. & PRE & Est. & PRE & Est. & PRE \\
\hline $\mathfrak{z}$ R.rss & 442.45 & $\hat{\mathfrak{z}}$ D.rss & 100 & $\hat{\mathfrak{z}}_{k k . r s s 5}$ & 933.63 \\
\hline$\hat{\mathfrak{z}} S D . r s s$ & 443.82 & $\hat{\mathfrak{z}} k k . r s s 1$ & 563.25 & $\hat{\mathfrak{z}}_{k k . r s s 6}$ & 791.95 \\
\hline$\hat{\mathfrak{z}}_{S K . r s s}$ & 447.46 & $\hat{\mathfrak{z}}_{k k . r s s 2}$ & 564.66 & $\hat{\mathfrak{z}}_{k k . r s s 7}$ & 564.17 \\
\hline$\hat{\mathfrak{z}} U P . r s s 1$ & 446.51 & $\hat{\mathfrak{z}}_{k k . r s s} 3$ & 563.56 & $\hat{\mathfrak{z}}_{k k . r s s} 8$ & 568.39 \\
\hline$\hat{\mathfrak{z}} U$ P.rss 2 & 442.76 & $\hat{\mathfrak{z}} k k . r s s 4$ & 567.41 & $\hat{\mathfrak{z}}_{N}$ & 1093.47 \\
\hline
\end{tabular}

\section{Conclusion}

In this article we have suggested regression-type estimator in ranked set sampling and extended our work for the case of scramble response. The results of 
TABLE 5. PREs for sensitive study variate using Eichhorn and Hayre model (1983)

\begin{tabular}{|c|c|c|c|c|c|}
\hline Est. & PRE & Est. & PRE & Est. & PRE \\
\hline$\hat{\mathfrak{z}}$ R.rss & 496.82 & $\hat{\mathfrak{z}}_{D . r s s}$ & 100 & $\hat{\mathfrak{z}}_{k k . r s s 5}$ & 2340.71 \\
\hline$\hat{\mathfrak{z}}_{S D . r s s}$ & 501.36 & $\hat{\mathfrak{z}}_{k k . r s s 1}$ & 641.99 & $\hat{\mathfrak{z}}_{k k . r s s} 6$ & 1647.31 \\
\hline$\hat{\mathfrak{z}} S K . r s s$ & 513.45 & $\hat{\mathfrak{z}}_{k k . r s s} 2$ & 647.54 & $\hat{\mathfrak{z}} k k . r s s 7$ & 644.98 \\
\hline$\hat{\mathfrak{z}}_{U P . r s s} 1$ & 510.29 & $\hat{\mathfrak{z}}_{k k . r s s} 3$ & 643.21 & $\hat{\mathfrak{z}}_{k k} k . r s s 8$ & 662.28 \\
\hline$\hat{\mathfrak{z}} U P . r s s 2$ & 497.82 & $\hat{\mathfrak{z}}_{k k . r s s 4}$ & 658.42 & $\hat{\mathfrak{z}}_{N}$ & 4812.21 \\
\hline
\end{tabular}

TABle 6. PREs for sensitive study variate using Saha model (2007)

\begin{tabular}{|c|c|c|c|c|c|}
\hline Est. & PRE & Est. & PRE & Est. & PRE \\
\hline$\hat{\hat{\mathfrak{z}}}$ R.rss & 486.44 & $\hat{\hat{\mathfrak{z}}}$ D.rss & 100 & $\hat{\mathfrak{z}}_{k k . r s s 5}$ & 1597.63 \\
\hline$\hat{\mathfrak{z}}_{S D}$.rss & 489.29 & $\hat{\mathfrak{z}}_{k k . r s s 1}$ & 615.62 & $\hat{\mathfrak{z}} k k . r s s 6$ & 1202.69 \\
\hline$\hat{\mathfrak{z}}_{S K}$.rss & 496.88 & $\hat{\mathfrak{z}}_{k k . r s s 2}$ & 618.93 & $\hat{\mathfrak{z}}_{k k . r s s 7}$ & 617.56 \\
\hline$\hat{\mathfrak{z}} U$ P.rss 1 & 494.89 & $\hat{\mathfrak{z}}_{k k . r s s 3}$ & 616.35 & $\hat{\mathfrak{z}}_{k k . r s s 8}$ & 627.72 \\
\hline$\hat{\mathfrak{z}} U P . r s s 2$ & 487.07 & $\hat{\mathfrak{z}} k k . r s s 4$ & 625.42 & $\hat{\mathfrak{z}}_{N}$ & 2653.81 \\
\hline
\end{tabular}

TABle 7. PREs for sensitive study variate using Diana and Perri model (2010)

\begin{tabular}{|c|c|c|c|}
\hline Est. & $\begin{array}{l}\phi=0.25 \\
\text { PRE }\end{array}$ & $\begin{array}{l}\phi=0.50 \\
\text { PRE }\end{array}$ & $\begin{array}{l}\phi=0.75 \\
\text { PRE }\end{array}$ \\
\hline$\hat{\mathfrak{z}}$ R.rss & 515.48 & 490.26 & 519.88 \\
\hline$\hat{\mathfrak{z}}_{S D . r s s}$ & 518.57 & 492.45 & 521.80 \\
\hline$\hat{\mathfrak{z}}_{S K . r s s}$ & 526.79 & 498.25 & 526.89 \\
\hline$\hat{\mathfrak{z}} U P 1 . r s s$ & 524.64 & 496.73 & 525.56 \\
\hline$\hat{\mathfrak{z}} U P 2 . r s s$ & 516.17 & 490.75 & 520.31 \\
\hline$\hat{\mathfrak{z}}$ D.rss & 100 & 100 & 100 \\
\hline$\hat{\mathfrak{z}}_{k k . r s s 1}$ & 651.80 & 607.29 & 639.74 \\
\hline$\hat{\mathfrak{z}}_{k k . r s s 2}$ & 655.39 & 609.70 & 641.76 \\
\hline$\hat{\mathfrak{z}}_{k k . r s s 3}$ & 652.59 & 607.82 & 640.19 \\
\hline$\hat{\mathfrak{z}}_{k k . r s s 4}$ & 662.42 & 614.41 & 645.72 \\
\hline$\hat{\mathfrak{z}}_{k k . r s s 5}$ & 1711.18 & 1298.61 & 1194.75 \\
\hline$\hat{\mathfrak{z}}_{k k . r s s 6}$ & 1285.40 & 1023.71 & 977.52 \\
\hline$\hat{\mathfrak{z}}_{k k . r s s 7}$ & 653.90 & 608.80 & 641.07 \\
\hline$\hat{\mathfrak{z}}_{k k . r s s 8}$ & 664.91 & 616.08 & 647.12 \\
\hline$\hat{\mathfrak{z}}_{N}$ & 2866.17 & 1825.57 & 1465.23 \\
\hline
\end{tabular}

the simulation study, available in Table $[3,4,5,6 \& 7]$, show the superiority of suggested estimator over Kadilar et al. [5, Koyuncu and Kadilar [7] and Mehta and 
Mandowara [9] estimators. Hence, it is recommended to utilize the suggested estimator.

\section{REFERENCES}

[1] Bouza, C. N., Ranked set sampling for the product estimator, Rev. Invest. Oper. 29(3), (2008), 201-206.

[2] Diana, G. and Perri, P.F., New scrambled response model for estimating the mean of a sensitive quantitative character, Journal of Applied Statistics 37,(2010), 1875-1890.

[3] Eichhorn, B. and Hayre, L.S., Scrambled randomized response method for obtaining sensitive quantitative data, Journal of Statistical Planning and Inference 7, (1983), 307-316.

[4] Gupta, S., Shabbir, J., Sousa, R. and Corte-Real, P., Estimation of the mean of a sensitive variable in the presence of auxiliary information, Communications in Statistics-Theory and Methods 41, (2012), 2394-2404.

[5] Kadilar,C., Unyazici,Y. and Cingi,H., Ratio estimator for the population mean using ranked set sampling, Statist. Papers 50, (2009), 301-309.

[6] Koyuncu, N., Gupta, S. and Sousa, R., Exponential-type estimators of the mean of a sensitive variable in the presence of nonsensitive auxiliary information, Communications in Statistics - Simulation and Computation 43(7), (2014), 1583-1594.

[7] Koyuncu, N. and Kadilar, C., Efficient estimators for the population mean, Hacett. J. Math. Statist. 38(2),(2009), 217-225.

[8] McIntyre, G.A., A method for unbiased selective sampling using ranked sets, Australian Journal of Agricultural Research 3, (1952), 385-390.

[9] Mehta, N. and Mandowara, V., A modified ratio-cum-product estimator of finite population mean using ranked set sampling, Communications in Statistics-Theory and Methods 45(2), (2016), 267-276.

[10] Pollock, K.H. and Bek, Y., A comparison of three randomized response models for quantitative data, Journal of the American Statistical Association 71,(1976), 884-886.

[11] Saha, A., A randomized response technique for quantitative data under unequal probability sampling, Journal of Statistical Theory and Practice 2(4),(2008), 589-596.

[12] Samawi, H. M. and Muttlak, H. A., Estimation of ratio using rank set sampling, Biomet. J. 38, (1996), 753-764.

[13] Shahzad, U., On the estimation of population mean under systematic sampling using auxiliary attributes, Orient J Phys Sciences 1, (2016), 17-22.

[14] Singh, H. P. and Kakran M. S., A modified ratio estimator using known coefficient of kurtosis of an auxiliary character, In Singh, S. ed. Advanced Sampling Theory with Applications, Kluwer Academic Publishers,(1993).

[15] Singh, H.P., Tailor, R. and Singh, S., General procedure for estimating the population mean using ranked set sampling, Journal of Statistical Computation and Simulation 84(5), (2014), 931-945.

[16] Singh, S., Advanced Sampling Theory with Applications. Vol. 1 and 2. London: Kluwer Academic Publishers, (2003).

[17] Sisodia, B. V. S. and Dwivedi, V. K., A modified ratio estimator using coefficient of variation of auxiliary variable, J. Ind. Soc. Agri. Statist. 33, (1981), 13-18.

[18] Sousa, R., Shabbir, J., Corte-Real, P., Gupta, S. , Ratio estimation of the mean of a sensitive variable in the presence of auxiliary information, Journal of Statistical Theory and Practice $4(3),(2010), 495-507$.

[19] Tailor, R., Sharma, B., A modified ratio-cum-product estimator of finite population mean using known coefficient of variation and coefficient of kurtosis. Stat. Transition New Ser. $10(1),(2009), 15-24$. 
[20] Upadhyaya, L. N., Singh, H. P., Use of transformed auxiliary variable in estimating the finite population mean, Biometrical J. 41, (1999), 627-636.

Current address: Usman Shahzad: Department of Mathematics and Statistics,PMAS Arid Agriculture University Rawalpindi, Pakistan.

E-mail address: usman.stat@yahoo.com

ORCID Address: https://orcid.org/0000-0002-0178-5298

Current address: Muhammad Hanif: Department of Mathematics and Statistics,PMAS Arid Agriculture University Rawalpindi, Pakistan.

E-mail address: mhpuno@hotmail.com

ORCID Address: https://orcid.org/0000-0002-1976-4452

Current address: Nursel Koyuncu: Department of Statistics, University of Hacettepe, Ankara, Turkey.

E-mail address: nkoyuncu@hacettepe.edu.tr

ORCID Address: https://orcid.org/0000-0003-1065-3411

Current address: Amelia Victoria Garcia Luengo: Department of Mathematics, University of Almeria, Spain.

E-mail address: amgarcia@ual.es

ORCID Address: https://orcid.org/0000-0001-7921-8384 